\section{A UTILIZAÇÃO DE CONTRACEPTIVOS HORMONAIS POR ADOLESCENTES E POTENCIAIS RISCOS PARA A SAÚDE}

\section{THE USE OF HORMONAL CONTRACEPTIVES BY ADOLESCENTS AND POTENTIAL HEALTH RISKS}

\author{
Lucélia Caroline dos Santos Cardoso ${ }^{1}$, André Luis Bendl ${ }^{1}$, \\ Luzia Teresinha Vianna dos Santos ${ }^{2}$, Bruna Lais de Oliveira Lima ${ }^{3}$, \\ Michele Einloft ${ }^{3}$, Andressa Souza $^{1}$
}

\section{RESUMO}

Reunir informações referentes aos riscos do uso de contraceptivos hormonais por adolescentes. Revisão integrativa da literatura científica dos últimos 10 anos. Incluídos os estudos sobre uso de anticoncepcional por adolescentes e os riscos associados. Estratégia de busca baseou-se na combinação dos descritores "adolescente", "saúde do adolescente", "anticoncepção", "fatores de risco" e "efeitos adversos" e seus respectivos mesh terms resultando em 73 artigos. Aplicando-se critérios de exclusão, restaram 22 artigos que foram analisados e agrupados em quadros. A literatura nacional e internacional menciona os métodos contraceptivos hormonais como seguros para uso por adolescentes porém ressaltam riscos cardiovasculares, alterações em gestações futuras e prejuízos ao sistema osteomuscular observados nas amostras estudadas, entre outros. A utilização de contracepção hormonal em adolescentes necessita de atenção e manejo cuidadoso para avaliação dos riscos e benefícios. Mais estudos abordando essa temática devem ser realizados para melhor esclarecimento sobre o assunto.

Palavras-chave: Adolescente; saúde do adolescente; anticoncepção; fatores de risco

\section{ABSTRACT}

To gather information related to the risks of the use of hormonal contraceptives by teenagers. An integrative review of the scientific literature of the past 10 years. Studies investigating the use of contraceptives by teenagers and the associated risks were included. Search strategy was based on the combination of descriptors "teen", "teen health", "contraception", "risk factors" and "adverse effects" and their respective mesh terms, resulting in 73 articles. After exclusion criteria were applied, 22 articles remained to be analyzed, and their data were grouped into charts. The national and international literatures consider hormonal contraceptive methods to be safe for use by teenagers, but mention cardiovascular risks, changes in future pregnancies and damages to the musculoskeletal system, among others, as potential complications observed in the samples. The use of hormonal contraception in adolescents requires attention and careful management to assess risks and benefits. Further studies should be conducted to clarify this topic.

Keywords: Adolescent; adolescent health; contraception; risk factors

Dados da Organização Mundial da Saúde (OMS) referem a adolescência como o período de vida entre 10 e 19 anos. Compreende uma etapa de transição entre a infância e fase adulta do indivíduo, momento caracterizado por impulsos de desenvolvimento físico, mental, emocional, social e sexual. Estimativas atuais projetam uma população adolescente de 1,2 milhões em nível mundial ${ }^{1}$.

A sexualidade está presente na vida dos adolescentes, cada vez mais precocemente. É importante e garantido aos adolescentes o direito de acesso à informações, educação e acesso aos métodos contraceptivos, bem como participação ativa na escolha do método de proteção, sendo garantido o acesso desses jovens ao sistema de saúde para orientação de tais medidas ${ }^{2,3}$ estando inclusive orientado pela $\mathrm{OMS}^{1}$ que os governos estruturem atendimento de
Clin Biomed Res. 2019;39(1):75-84

1 Programa de Pós-graduação em Saúde e Desenvolvimento Humano, Universidade La Salle (UNILASALLE). Canoas, RS, Brasil.

2 Faculdade de Enfermagem, Universidade Luterana do Brasil (ULBRA). Gravataí, RS, Brasil.

3 Unidade de Internação Clínica, Hospital de Clínicas de Porto Alegre (HCPA) Porto Alegre, RS, Brasil.

Autor correspondente: Andressa Souza andressasz@gmail.com Universidade La Salle (UNILASALLE) Av. Vítor Barreto, 2288. 92010-000, Canoas, RS, Brasil. 
qualidade à essa população. A relevância dessa informação torna-se ainda maior ao se considerar que as complicações decorrentes de gestação e parto em adolescentes são a segunda maior causa de morte entre meninas nessa faixa etária ${ }^{1}$. A importância dos anticoncepcionais é inegável, visto a diminuição significativa das taxas de mortalidade materna nos países em desenvolvimento, somente pela redução das gestações não desejadas ${ }^{4}$. Apesar de benéfico no ponto de vista dos indicadores de mortalidade materna, o uso de anticoncepcionais hormonais está associado a riscos que devem ser considerados no momento da sua escolha ${ }^{5}$.

A prescrição de anticoncepcionais é um ato médico que deve ser avaliado criteriosamente, sobretudo em menores de 14 anos. Deve-se avaliar ocorrência de violência sexual e documentar a adoção do método escolhido em conjunto com a paciente. A orientação é de que a maioria dos métodos disponíveis pode ser utilizada por adolescentes de acordo com critérios clínicos, mas que, alguns são mais adequados em outras fases do ciclo de vida das mulheres ${ }^{2,6}$, o que será observado e avaliado através de criteriosa anamnese e exame físico que descartem a presença de contraindicações ao uso ${ }^{5}$.

Estima-se que em torno de 140 milhões de mulheres façam uso de anticoncepcionais hormonais em todo mundo. Para o Brasil, a orientação geral dos cadernos de orientações do Ministério da saúde do Brasil $^{2}$ é que não há restrição ao uso dos anticoncepcionais hormonais na adolescência, sendo informado que os anticoncepcionais combinados de estrógeno e progesterona podem ser utilizados desde a menarca em suas diversas apresentações. Todavia, é orientado prudência na prescrição dos métodos progestágenos. Tais contraceptivos devem ser evitados em menores de 18 anos devido ao risco de diminuição da calcificação óssea decorrente do bloqueio do eixo hipotálamo-hipófise-ovário, causador de supressão na produção de estrogênio². Anticoncepcionais hormonais de baixa dosagem devem ser os de escolha para a população adolescente, tendo o componente estrogênico uma dosagem entre 15 e $35 \mu \mathrm{g}$ associado ao componente progestágeno de escolha ${ }^{5}$. Deve-se orientar o uso de preservativo e contracepção de emergência havendo necessidade, mas que a adolescente pode optar em qualquer etapa do seu desenvolvimento a escolha de método anticoncepcional, mantendo o uso de preservativos para proteção de doenças sexualmente transmissíveis ${ }^{3}$.

As dúvidas na prática clínica associadas ao reduzido contingente de estudos que abordem a temática dos riscos do uso de contraceptivos na população exclusivamente adolescente motiva a elaboração da proposta de revisão da literatura atual que possibilite a resolução do questionamento inicial quanto aos riscos a que essa população está exposta a curto e longo prazo. O presente artigo tem por objetivo apresentar informações referentes aos riscos do uso de contraceptivos hormonais por adolescentes.

\section{MATERIAL E MÉTODOS}

Trata-se de um estudo descritivo de revisão integrativa da literatura nacional e internacional recente referente ao risco de uso de anticoncepcionais por adolescentes. Visando buscar as mais atualizadas evidências científicas, pesquisou-se por artigos científicos indexados nas bases de dados PubMed, Bireme, Scielo e Lilacs. A estratégia de busca realizada foi estruturada para a inclusão dos artigos publicados nos últimos 10 anos (2006 a 2016) e disponíveis integralmente nas bases consultadas. Para realização da revisão foram utilizados os seguintes descritores: "adolescente", "saúde do adolescente", "anticoncepção", "fatores de risco" e "efeitos adversos" combinados entre si e com os mesh terms respectivos em língua inglesa "adolescent", "adolescent health", "contraception", "risk factors" e "adverse effects" Os descritores escolhidos e utilizados nas bibliotecas virtuais foram determinados com base nos Descritores em Ciências da Saúde (DeCS) sendo localizados inicialmente 73 artigos que contemplavam os dados descritos.

A apresentação dos dados encontrados baseou-se na estrutura do Preferred Reporting Items for Systematic Reviews and Meta-Analyses (PRISMA) e pode ser analisada na Figura 1. Foram excluídos

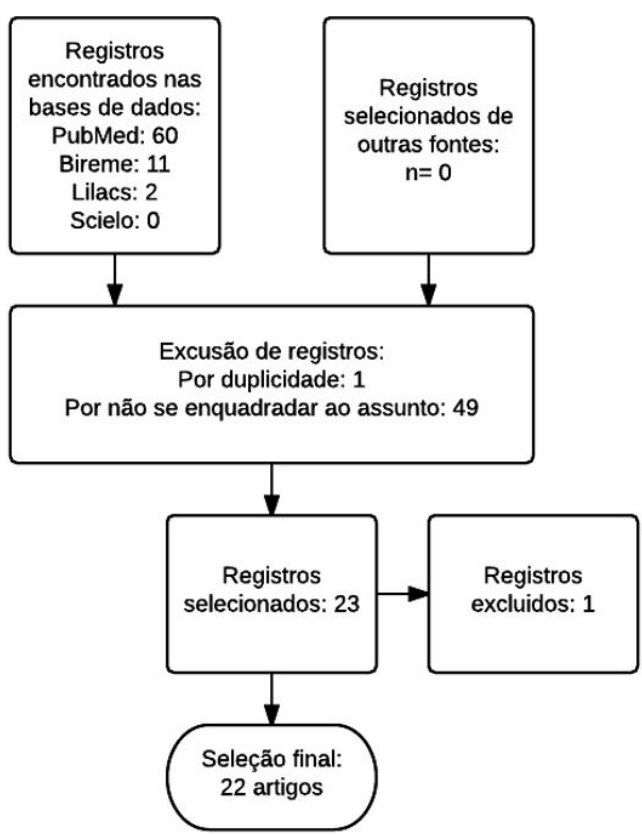

Figura 1: Fluxograma de realização da pesquisa baseada no PRISMA. 
os artigos que claramente não se enquadravam no tema proposto para a revisão, os artigos repetidos e aqueles que foram oriundos de pesquisas realizadas sem avaliação de adolescentes em sua amostra.

A presente revisão contou com critérios de seleção e elegibilidade de estudos. Quanto ao tipo de estudos, não houve seleção por delineamento específico, sendo considerado todos os tipos de estudo. Quanto aos participantes, foram selecionados os artigos que apresentaram dados de pesquisa que envolvessem adolescentes na amostra. Acerca das intervenções estudadas, foram selecionados os artigos que se referiam ao uso de anticoncepcionais e os ricos associados a curto e longo prazo.

Os dados coletados dos artigos selecionados foram organizados em quadros, onde, de forma qualitativa, suas principais informações foram descritas (autores e ano do estudo, características da população, objetivo do trabalho, resumo dos métodos e conclusões).

\section{RESULTADOS E DISCUSSÃO}

Nenhum artigo em português compõem a presente revisão, restringindo-se os dados à literatura internacional apesar do uso de descritores que contemplassem o idioma para a realização da busca nas bases de dados. Características populacionais e econômicas aparecem como notórias na realização de estudos europeus, onde a realização de coortes significativamente relevantes quanto ao assunto são apresentadas e devem ser consideradas, apesar das distinções entre as populações em estudo. O Quadro 1 apresenta a listagem dos artigos que compuseram o estudo, bem como seus respectivos autores, ano de publicação e nível de evidência de acordo com a Escala de Oxford.

Os resultados encontrados na literatura científica acerca dos riscos relacionados ao uso de métodos contraceptivos hormonais por adolescentes são apresentados distinguindo-os por métodos orais, injetáveis, uso de adesivos ou anel vaginal e utilização de dispositivo intra-uterino combinado ao hormônio. Salienta-se ainda na literatura as diferenças entre os métodos combinados e utilização de progetágenos isolados por adolescentes, tanto da forma oral como injetável, descrita a seguir.

O método contraceptivo reversível mais conhecido e utilizado no Brasil são os anticoncepcionais conjugados orais (ACO). Trata-se de medicação onde associam-se dois hormônios sintéticos, estrogênio e progestogênio, semelhantes aos produzidos pelos ovários ${ }^{2,6}$. Definições que norteiam o uso dos ACO em adolescentes consideram que, por ser um método

Quadro 1: Níveis de Evidência dos artigos pesquisados.

\begin{tabular}{|c|c|c|c|}
\hline Artigo & Autor/Ano & Delineamento & $\begin{array}{l}\text { Nível de } \\
\text { Evidência }\end{array}$ \\
\hline $\begin{array}{l}\text { Hormonal contraception increases the risk of psychotropic drug use } \\
\text { in adolescent girls but not in adults: A pharmacoepidemiological } \\
\text { study on } 800000 \text { Swedish women }\end{array}$ & $\begin{array}{l}\text { Zettermark et al. } \\
\qquad(2018)^{7}\end{array}$ & coorte & $2 \mathrm{~B}$ \\
\hline $\begin{array}{l}\text { Contemporary Hormonal Contraception and the Risk of Breast } \\
\text { Cancer }\end{array}$ & $\begin{array}{l}\text { Morch et al. } \\
(2018)^{8}\end{array}$ & coorte & $2 \mathrm{~B}$ \\
\hline $\begin{array}{l}\text { Nonoral combined hormonal contraceptives and thromboembolism: } \\
\text { a systematic review. }\end{array}$ & $\begin{array}{l}\text { Tepper et al. } \\
(2017)^{9}\end{array}$ & $\begin{array}{l}\text { Revisão } \\
\text { sistemática }\end{array}$ & $1 \mathrm{~A}$ \\
\hline $\begin{array}{l}\text { Pre-gravid oral contraceptive use in relation to birth weight: } \\
\text { a prospective cohort study. }\end{array}$ & $\begin{array}{l}\text { Hatch et al, } \\
(2015)^{10}\end{array}$ & coorte & $2 \mathrm{~B}$ \\
\hline $\begin{array}{l}\text { Positive Testing for Neisseria gonorrhea and Chlamydia trachomatis } \\
\text { and the Risk of Pelvic Inflammatory Disease in IUD Users. }\end{array}$ & $\begin{array}{l}\text { Birgisson et al. } \\
(2015)^{11}\end{array}$ & coorte & $2 \mathrm{~B}$ \\
\hline $\begin{array}{l}\text { Association between levonorgestrel emergency contraception and } \\
\text { the risk of ectopic pregnancy: a multicenter case-control study. }\end{array}$ & $\begin{array}{l}\text { Zhang et al. } \\
\qquad(2015)^{12}\end{array}$ & caso-controle & $3 B$ \\
\hline $\begin{array}{l}\text { Hormonal contraception and the risk of HIV acquisition: } \\
\text { an individual participant data meta-analysis. }\end{array}$ & $\begin{array}{l}\text { Morrison et al. } \\
(2015)^{13}\end{array}$ & Metanalise & $1 \mathrm{~A}$ \\
\hline $\begin{array}{l}\text { Risk of uterine perforation with levonorgestrel-releasing and copper } \\
\text { intrauterine devices in the European Active Surveillance Study on } \\
\text { Intrauterine Devices. }\end{array}$ & $\begin{array}{l}\text { Heinemann et al. } \\
\qquad(2015)^{14}\end{array}$ & coorte & $2 \mathrm{~B}$ \\
\hline $\begin{array}{l}\text { Monocyte CD40 expression in young healthy female smokers } \\
\text { and/or oral contraceptives users without additional risk factors for } \\
\text { atherosclerosis }\end{array}$ & $\begin{array}{l}\text { Parahuleva et al. } \\
\qquad(2015)^{15}\end{array}$ & caso-controle & $3 B$ \\
\hline $\begin{array}{l}\text { Hormonal contraceptive use before and after conception in relation } \\
\text { to preterm birth and small for gestational age: an observational } \\
\text { cohort study. }\end{array}$ & $\begin{array}{c}\text { Jensen et al. } \\
(2015)^{16}\end{array}$ & Coorte & $2 \mathrm{~B}$ \\
\hline
\end{tabular}


Quadro 1: Continuação...

\begin{tabular}{|c|c|c|c|}
\hline Artigo & Autor/Ano & Delineamento & $\begin{array}{l}\text { Nível de } \\
\text { Evidência }\end{array}$ \\
\hline $\begin{array}{l}\text { The association between contraceptive use at the time of } \\
\text { conception and hypertensive disorders during pregnancy: } \\
\text { a retrospective cohort study of prams participants. }\end{array}$ & $\begin{array}{l}\text { Farley et al. } \\
\qquad(2014)^{17}\end{array}$ & Coorte & 2B \\
\hline $\begin{array}{l}\text { Use of depot medroxyprogesterone acetate contraception and } \\
\text { incidence of bone fracture. }\end{array}$ & $\begin{array}{l}\text { Lanza et al, } \\
(2013)^{18}\end{array}$ & coorte & $2 \mathrm{~B}$ \\
\hline $\begin{array}{l}\text { Combined oral contraceptives' influence on weight, body } \\
\text { composition, height, and bone mineral density in girls younger than } \\
18 \text { years: a systematic review. }\end{array}$ & $\begin{array}{l}\text { Warholm et al, } \\
(2012)^{19}\end{array}$ & $\begin{array}{l}\text { revisao } \\
\text { sistemática }\end{array}$ & $1 \mathrm{~A}$ \\
\hline $\begin{array}{l}\text { Higher risk of venous thrombosis associated with drospirenone- } \\
\text { containing oral contraceptives: a population-based cohort study. }\end{array}$ & $\begin{array}{l}\text { Gronich et al. } \\
(2011)^{20}\end{array}$ & Coorte & 2B \\
\hline $\begin{array}{l}\text { Hormonal contraception and thrombotic risk: a multidisciplinary } \\
\text { approach. }\end{array}$ & $\begin{array}{l}\text { Trenor et al. } \\
(2011)^{21}\end{array}$ & $\begin{array}{l}\text { revisao de } \\
\text { literatura }\end{array}$ & $1 \mathrm{~A}$ \\
\hline $\begin{array}{l}\text { Hormonal contraceptives and the length of their use are not } \\
\text { independent risk factors for high-risk HPV infections or high-grade } \\
\text { CIN. }\end{array}$ & $\begin{array}{l}\text { Longatto- } \\
\text { Filho et al. } \\
(2011)^{22}\end{array}$ & Coorte & 2B \\
\hline $\begin{array}{l}\text { Use of oral contraceptives containing gestodene and risk of venous } \\
\text { thromboembolism: outlook } 10 \text { years after the third-generation } \\
\text { "pill scare". }\end{array}$ & $\begin{array}{l}\text { Heinemann et al. } \\
\qquad(2010)^{23}\end{array}$ & caso-controle & 3B \\
\hline $\begin{array}{l}\text { Hormonal contraception, sickle cell trait, and risk for venous } \\
\text { thromboembolism among African American women. }\end{array}$ & $\begin{array}{l}\text { Austin et al. } \\
(2009)^{24}\end{array}$ & caso-controle & 3B \\
\hline Maternal use of oral contraceptives and risk of fetal death. & $\begin{array}{l}\text { Jellesen et al. } \\
(2008)^{25}\end{array}$ & Coorte & 2B \\
\hline $\begin{array}{l}\text { Pre-gravid oral contraceptive use in relation to birth weight: } \\
\text { a prospective cohort study. }\end{array}$ & $\begin{array}{l}\text { Hatch et al. } \\
(2015)^{10}\end{array}$ & coorte & 2B \\
\hline $\begin{array}{l}\text { Positive Testing for Neisseria gonorrhea and Chlamydia trachomatis } \\
\text { and the Risk of Pelvic Inflammatory Disease in IUD Users. }\end{array}$ & $\begin{array}{l}\text { Birgisson et al. } \\
(2015)^{11}\end{array}$ & coorte & 2B \\
\hline
\end{tabular}

muito conhecido, faz com que essa seja uma possível razão de escolha para pacientes adolescentes ${ }^{5}$. Trata-se de medida vantajosa no sentido de proteção para anemia, cistos foliculares, tumores benignos de mama e ovários, câncer de ovários, endométrio e colo retal, diminuição dos sintomas da dismenorreia além de seu uso poder ser iniciado na adolescência e findar-se com a menopausa sem a necessidade de interrupção. Concomitantemente, sua utilização está associada à complicações sérias como trombose venosa profunda e contraindicada para pacientes que possuam histórico atual ou pregresso de eventos tromboembólicos, certeza ou suspeita de câncer de mama e outros tumores hormônio-dependentes, hepatopatia aguda ou crônica, hipertensão arterial, doença vascular, enxaqueca com sintomas neurológicos focais, diabetes com complicações e lúpus. Efeitos colaterais frequentes são as náuseas, sangramento vaginal irregular, cefaleia, hipersensibilidade mamária e alterações no humor. O cloasma e aumento da pressão arterial associado ao consumo de ACO constam nos manuais ministeriais brasileiro².

Conforme observado na revisão realizada, outros autores destacam o risco para complicações do sistema vascular associado ao uso de anticoncepcionais hormonais. Destaque especial se dá aos anticoncepcionais hormonais combinados com o componente drospiredona, muito utilizado pela população adolescente. Avaliação e aconselhamento individualizadas de acordo com o risco cardiovascular apresentado pelas pacientes são de extrema importância, haja visto a exposição a maiores fatores de risco por esse grupo ${ }^{26}$.

O Quadro 2 apresenta os dados da literatura pesquisada referentes às complicações do sistema cardiovascular associadas ao uso de anticoncepcionais observadas nos esudos realizados com a população adolescente e adulta. Nenhum estudo encontrado com a estratégia de busca utilizada foi realizado exclusivamente com adolescentes, tendo os resultados dispostos sobre a população adulta estudada em conjunto com o grupo de interesse da atual revisão e representados a seguir.

Em decorrência da possibilidade de complicações, recomenda-se que as adolescentes que devem submeter-se a cirurgias em membros inferiores ou que necessitem de imobilização pós-operatória devem cessar o uso de ACO pelo risco aumentado de trombose venosa profunda e edema pulmonar ${ }^{5}$. Além disso, é salientado que existe risco de acidente 
Quadro 2: Complicações sobre o sistema cardiovascular associado ao uso de anticoncepcional hormonal.

\begin{tabular}{|c|c|c|c|c|}
\hline Autor/Ano & $\begin{array}{c}\text { Característica da } \\
\text { população }\end{array}$ & Objetivos & Delineamento & Conclusões \\
\hline $\begin{array}{c}\text { Tepper et al. } \\
(2017)^{9}\end{array}$ & & \begin{tabular}{|c|} 
Examinar o risco de \\
tromboembolismo \\
venoso e arterial entre \\
as mulheres que \\
usaram contraceptivos \\
hormonais orais em \\
comparação com as \\
mulheres que não usam \\
hormonais orais
\end{tabular} & Revisão sistemática & $\begin{array}{l}\text { Resultados não são } \\
\text { conclusivos quanto à } \\
\text { diferenciação dos riscos } \\
\text { entre o uso de métodos } \\
\text { contraceptivos orais e } \\
\text { não orais necessitando } \\
\text { de maiores análises. }\end{array}$ \\
\hline $\begin{array}{l}\text { Gronich et al. } \\
(2011)^{20}\end{array}$ & $\begin{array}{l}\text { Todas as mulheres } \\
\text { de } 12 \text { a } 50 \text { anos que } \\
\text { tiveram prescrição } \\
\text { de anticoncepcional } \\
\text { oral combinado nesse } \\
\text { período em Israel }\end{array}$ & \begin{tabular}{|c|} 
Avaliar o uso da \\
drospirenona à \\
possível associação \\
com aumento do risco \\
de trombose quando \\
comparada com outros \\
anticoncepcionais orais.
\end{tabular} & Coorte & $\begin{array}{l}\text { O uso da drospirenona } \\
\text { foi associado ao } \\
\text { aumento do risco de } \\
\text { trombose venosa } \\
\text { profunda e embolia } \\
\text { pulmonar, além de } \\
\text { acidente vascular } \\
\text { cerebral. }\end{array}$ \\
\hline $\begin{array}{c}\text { Trenor et al. } \\
(2011)^{21}\end{array}$ & $\begin{array}{l}\text { Estudos publicados e } \\
\text { revisados conforme } \\
\text { estratégia de busca }\end{array}$ & $\begin{array}{l}\text { Revisar os riscos de } \\
\text { eventos trombóticos em } \\
\text { usuárias de métodos } \\
\text { anticoncepcionais em } \\
\text { adolescentes }\end{array}$ & Revisão de literatura & $\begin{array}{l}\text { Foi observado aumento } \\
\text { de } 3 \text { a } 5 \text { vezes o risco } \\
\text { de eventos trombóticos } \\
\text { entre adolescentes } \\
\text { usuárias de ACO sendo } \\
\text { o risco normal desse } \\
\text { evento em adolescente } \\
\text { não usuárias de } \\
\text { ACO de } 0,05 \% \text {. } \\
\text { Anticoncepcionais } \\
\text { somente de } \\
\text { progestágeno e os de } \\
\text { estradiol transdérmico } \\
\text { não apresentaram } \\
\text { riscos ou foram } \\
\text { mínimos. Demais } \\
\text { métodos, os autores } \\
\text { sugerem que outros } \\
\text { estudos devem ser } \\
\text { realizados. }\end{array}$ \\
\hline $\begin{array}{c}\text { Austin et al. } \\
(2009)^{24}\end{array}$ & $\begin{array}{c}60 \text { mulheres africanas } \\
\text { com tromboembolia e } \\
196 \text { controles }\end{array}$ & $\begin{array}{l}\text { Avaliar o efeito do } \\
\text { uso de contraceptivos } \\
\text { orais hormonais e a } \\
\text { associação com risco } \\
\text { de tromboembolismo } \\
\text { entre as mulheres } \\
\text { afro-americanas e } \\
\text { possível intensificação } \\
\text { no traço falciforme }\end{array}$ & Caso-controle & $\begin{array}{c}\text { O uso de } \\
\text { anticoncepcional } \\
\text { hormonal aumenta } \\
\text { o risco de eventos } \\
\text { trombóticos na } \\
\text { amostra. Em mulheres } \\
\text { portadoras de traço } \\
\text { falciforme esse risco é } \\
\text { ainda maior. }\end{array}$ \\
\hline
\end{tabular}

vascular cerebral, infarto do miocárdio e trombose venosa profunda entre as usuárias do método, principalmente em tabagistas, independente da faixa etária. Tal dado é de grande importância, pois dados mundiais indicam que 1 a cada 10 adolescentes fazem uso de tabaco, sendo que em algumas localidades esse número pode ser ainda maior ${ }^{1}$. Observa-se que tabagismo não é um único fator complicador isolado, mas sim, que pertence a um grupo de complicações, inclusive aumento da pressão arterial sistólica e diastólica entre as usuárias da amostra estudada ${ }^{26}$.

Dados quanto à utilização de anticoncepcionais hormonais e complicações em gestações futuras são contraditórios. Autores observaram a utilização de métodos anticoncepcionais hormonais à prematuridade $^{16}$ e ocorrência de baixo peso ao nascer ${ }^{10}$. Em contrapartida, estudo publicado em 2013 ressalta que não foram encontradas associações de 
seu uso com óbito fetal ${ }^{25}$ tampouco à complicações quanto a hipertensão gestacional, por exemplo ${ }^{17}$. A análise dos dados coletados na presente revisão a respeito do risco gestacional podem ser melhor observados na Quadro 3.

Por semelhança no quesito de combinações hormonais, os anticoncepcionais combinados injetáveis possuem as mesmas contraindicações e efeitos colaterais do método oral. À disposição de mercado estão fórmulas de aplicação mensal além de outros métodos com semelhantes indicações e adversidades, como o método transdérmico (adesivo) e o anel vaginal. Com essas opções, a liberação diária de hormônios combinados se dá por via transdérmica por adesivos que necessitam de trocas semanais ou por dispositivos vaginais. A opção transdérmica trata-se de um método onde a liberação diária de hormônios apresenta-se mais elevada que no uso oral, porém, não há comprovação de que essa característica aumenta o risco de tromboembolismo. Importante observar que, por suas especificidades de formulação, esse método apresenta-se menos eficaz em casos de obesidade ${ }^{5}$.

Os métodos hormonais com progestógeno isolado são recomendados para pacientes com contraindicações ao uso de estrógeno e lactentes. Trata-se de um método que exige maior precisão em controle de horários de consumo. A versão injetável trimestral possui restrições em pacientes adolescentes devido ao seu efeito sobre a densidade óssea. Além disso, trata-se de um método onde existe demora do retorno da fertilidade após a suspensão do uso ${ }^{5,6}$. É evidente na literatura pesquisada a questão do prejuízo sobre a densidade óssea em estudo realizado com adolescentes inclusive com anticoncepcionais combinados (etinilestradiol 20ug/desogestrel 150mg) 2 $^{27}$.

No Quadro 4 é possível observar questões referentes aos prejuízos e particularidades quanto às estruturas musculoesqueléticas sob ação de anticoncepcionais hormonais, principalmente medroxiprogesterona.

Quadro 3: Risco gestacional relacionado ao uso de anticoncepcional hormonal por adolescentes.

\begin{tabular}{|c|c|c|c|c|}
\hline Autor/Ano & $\begin{array}{c}\text { Característica da } \\
\text { população }\end{array}$ & Objetivos & Delineamento & Conclusões \\
\hline $\begin{array}{l}\text { Hatch et al. } \\
(2015)^{10}\end{array}$ & $\begin{array}{c}5901 \text { gestações } \\
\text { planejadas e } \\
4046 \text { nascidos vivos }\end{array}$ & $\begin{array}{l}\text { Observar o peso ao } \\
\text { nascer e o tempo de uso } \\
\text { de anticoncepcional pré } \\
\text { gestacional pela mãe }\end{array}$ & $\begin{array}{c}\text { Coorte } \\
\text { prospectivo }\end{array}$ & $\begin{array}{l}\text { O uso de ACO por longo } \\
\text { periodo de tempo pode estar } \\
\text { associado a baixo peso ao } \\
\text { nascimento, principalmente em } \\
\text { periodo maior que } 12 \text { meses }\end{array}$ \\
\hline $\begin{array}{l}\text { Jensen et al. } \\
(2015)^{16}\end{array}$ & $\begin{array}{l}44.734 \text { gestações } \\
\text { foram analisadas }\end{array}$ & \begin{tabular}{|c|} 
Avaliar se os \\
contraceptivos hormonais, \\
utilizados antes ou no \\
início da gravidez, pode \\
conferir um aumento \\
do risco de parto \\
prematuro ou redução do \\
crescimento fetal. \\
\end{tabular} & Coorte & $\begin{array}{c}\text { Observado relação positiva } \\
\text { entre partos prematuros e uso } \\
\text { prévio de anticoncepcional } \\
\text { hormonal oral em todos os } \\
\text { períodos de exposição. Maior } \\
\text { aumento nos casos onde } \\
\text { se utilizou noretisterona na } \\
\text { formulação. } \\
\end{array}$ \\
\hline $\begin{array}{l}\text { Zhang et al. } \\
\qquad(2015)^{12}\end{array}$ & $\begin{array}{l}2411 \text { Gestações } \\
\text { Ectópicas + } \\
2416 \text { gestações + } \\
2419 \text { controles }\end{array}$ & $\begin{array}{c}\text { Observar se o uso } \\
\text { de contracepção } \\
\text { de emergência está } \\
\text { associada ao aumento } \\
\text { de casos de gestação } \\
\text { ectópica } \\
\end{array}$ & Caso-controle & $\begin{array}{c}\text { O uso correto é seguro, porém } \\
\text { o uso de nova dosagem } \\
\text { no mesmo ciclo apresenta } \\
\text { aumento de casos de gestação } \\
\text { ectópica }\end{array}$ \\
\hline $\begin{array}{l}\text { Farley et al. } \\
(2014)^{17}\end{array}$ & $\begin{array}{l}2395 \text { mulheres de } \\
18 \text { a } 45 \text { anos }\end{array}$ & $\begin{array}{l}\text { Examinar a associação } \\
\text { entre o uso de } \\
\text { contraceptivos no } \\
\text { momento da concepção } \\
\text { e complicações } \\
\text { hipertensivas durante a } \\
\text { gravidez. }\end{array}$ & Coorte & $\begin{array}{c}\text { Não foi encontrado forte } \\
\text { associação de métodos } \\
\text { contraceptivos e complicações } \\
\text { hipertensivas na gestação } \\
\text { (1,22 vezes maior do que } \\
\text { nas usuárias de método de } \\
\text { barreira). Sugere-se maiores } \\
\text { estudos sobre o assunto para } \\
\text { elucidação dos dados. } \\
\end{array}$ \\
\hline $\begin{array}{l}\text { Jellesen et al. } \\
\qquad(2008)^{25}\end{array}$ & 92.719 mulheres & $\begin{array}{l}\text { Avaliar se existe aumento } \\
\text { do número de mortes } \\
\text { fetais entre as usuárias de } \\
\text { anticoncepcionais }\end{array}$ & Coorte & $\begin{array}{c}\text { Não foram encontradas } \\
\text { associações entre uso de } \\
\text { anticoncepcional e ocorrência } \\
\text { de óbito fetal entre as } \\
\text { participantes }\end{array}$ \\
\hline
\end{tabular}


Já os implantes subdérmicos são citados como uma forma interessante de prevenção para gravidez para adolescentes que visem um método preventivo longo. Seu alto custo interfere na escolha, além da disposição dificultada pela exigência de profissional treinado para a colocação e retirada do implante ${ }^{5,6}$.

Também caracterizado como um método de longa duração, o dispositivo intra-uterino (DIU), apesar de pouco utilizado para adolescentes, tem relatos interessantes na literatura analisada. Os dados coletados sobre a utilização desse método podem ser observados no Quadro 5.

A literatura revisada apontou a contribuição de estudos que apontam segurança e não encontraram fortes associações de complicações na utilização de métodos hormonais através de estudos realizados em amostra de mulheres com idade variada. Dúvidas quanto a associação ou não à infecções sexualmente transmissíveis e infecções ginecológicas foram comparadas e descartadas através da realização de estudos por diferentes autores. Tais dados podem ser observados analisando-se o Quadro 6.

Evidencia-se a ocorrência de maior número de transtorno de ansiedade e depressão em mulheres e os hormônios sexuais femininos podem ter contribuição nesse padrão de diferenciação ${ }^{7}$. Tanto é perceptível essa preocupação que estudos vem sendo realizados a fim de comprovar ou descartar a associação do uso de métodos contraceptivos hormonais com efeitos adversos sobre a saúde psicológica das mulheres, a exemplo da Suécia onde um coorte maior que 800.000 participantes foram acompanhadas e comprovadas a associação, no público adolescente, de maior uso de drogas psicotrópicas após início da terapia com anticoncepcional hormonal, principalmente na população de 12 a 14 anos. O mesmo resultado não foi observado na população adulta, o que demostra a fragilidade e susceptibilidade do público

Quadro 4: Ação dos anticoncepcionais hormonais sobre sistema musculoesquelético.

\begin{tabular}{|c|c|c|c|c|}
\hline Autor/Ano & $\begin{array}{l}\text { Característica da } \\
\text { população }\end{array}$ & Objetivos & Delineamento & Conclusões \\
\hline $\begin{array}{l}\text { Lanza et al. } \\
\qquad(2013)^{18}\end{array}$ & $\begin{array}{c}312,395 \text { mulheres } \\
\text { e 11,822 casos de } \\
\text { fratura }\end{array}$ & $\begin{array}{c}\text { Determinar se o uso } \\
\text { de medroxiproesterona } \\
\text { injetável aumenta o risco } \\
\text { de fratura em usuárias }\end{array}$ & $\begin{array}{l}\text { Coorte } \\
\text { retrospectivo }\end{array}$ & $\begin{array}{c}\text { As usuárias de } \\
\text { medroxiprogesterona } \\
\text { tiveram maior número de } \\
\text { casos de fratura, mas não } \\
\text { encontrou-se associação do } \\
\text { uso da medicação aos casos } \\
\text { encontrados }\end{array}$ \\
\hline $\begin{array}{l}\text { Warholm et al. } \\
(2012)^{19}\end{array}$ & $\begin{array}{c}\text { Estudos publicados } \\
\text { em base de dados } \\
\text { de } 1990-2012\end{array}$ & $\begin{array}{l}\text { Investigar as evidências } \\
\text { das influências dos } \\
\text { ACO sobre peso, altura } \\
\text { e densidade óssea em } \\
\text { menores de } 18 \text { anos }\end{array}$ & $\begin{array}{c}\text { Revisão } \\
\text { sistemática }\end{array}$ & $\begin{array}{c}\text { Não foram encontradas } \\
\text { evidências de impacto } \\
\text { negativo dos ACO sobre peso } \\
\text { e altura nos poucos estudos } \\
\text { encontrados. Risco sobre a } \\
\text { densidade óssea não podem } \\
\text { ser descartados. OS autores } \\
\text { sugerem a necessidade de } \\
\text { mais estudos prospectivos a } \\
\text { esse respeito. }\end{array}$ \\
\hline
\end{tabular}

Quadro 5: Influência do uso de DIU por adolescentes.

\begin{tabular}{|c|c|c|c|c|}
\hline Autor/Ano & $\begin{array}{c}\text { Característica da } \\
\text { população }\end{array}$ & Objetivos & Delineamento & Conclusões \\
\hline $\begin{array}{c}\text { Birgisson et al. } \\
(2015)^{11}\end{array}$ & $\begin{array}{c}9000 \text { mulheres de } \\
14-45 \text { anos }\end{array}$ & $\begin{array}{c}\text { Observar risco de } \\
\text { doença pélvica em } \\
\text { usuárias de DIU }\end{array}$ & $\begin{array}{c}\text { O uso de DIU não amentou } \\
\text { o risco para doença pélvica } \\
\text { na população em estudo. } \\
\text { A população foi acompanhada } \\
\text { por período de } 2 \text { a 3 anos } \\
\text { após a colocação }\end{array}$ \\
\hline $\begin{array}{c}\text { Heinemann et al. } \\
(2015)^{14}\end{array}$ & 61.648 mulheres & $\begin{array}{c}\text { Observar o risco de } \\
\text { perfuração uterina } \\
\text { causada após a } \\
\text { inserção de DIU }\end{array}$ & $\begin{array}{c}\text { Coorte prospectiva } \\
\text { multinacional }\end{array}$ & $\begin{array}{c}\text { Baixo risco de perfuração } \\
\text { (81 casos relatados). Casos } \\
\text { encontrados principalmente } \\
\text { quando associado à } \\
\text { amamentação no momento } \\
\text { da inserção. }\end{array}$ \\
\hline
\end{tabular}


Quadro 6: Não associação de infecções sexualmente transmissíveis (ISTs) ao uso de métodos contraceptivos hormonais por adolescentes.

\begin{tabular}{|c|c|c|c|c|}
\hline Autor/Ano & $\begin{array}{l}\text { Característica } \\
\text { da população }\end{array}$ & Objetivos & Delineamento & Conclusões \\
\hline $\begin{array}{l}\text { Morrison et al. } \\
\qquad(2015)^{13}\end{array}$ & $\begin{array}{c}18 \text { estudos } \\
\text { incluindo } \\
37.124 \text { mulheres }\end{array}$ & $\begin{array}{l}\text { Observar se o uso de } \\
\text { métodos contraceptivos } \\
\text { aumenta o risco de } \\
\text { contaminação pelo } \\
\text { vírus HIV }\end{array}$ & Metanálise & \begin{tabular}{|c} 
Não comprova-se o risco mas \\
associa-se a possibilidade \\
de maior risco de contrair \\
HIV entre as usuárias \\
de medroxiprogesterona \\
injetável, sugerindo estudo \\
de um estudo clínico \\
randomizado para avaliação \\
desse desfecho
\end{tabular} \\
\hline $\begin{array}{c}\text { Farley et al. } \\
(2014)^{17}\end{array}$ & $\begin{array}{l}2395 \text { mulheres } \\
\text { de } 18 \text { a } 45 \text { anos }\end{array}$ & $\begin{array}{c}\text { Examinar a associação } \\
\text { entre o uso de } \\
\text { contraceptivos no } \\
\text { momento da concepção e } \\
\text { complicações hipertensivas } \\
\text { durante a gravidez. }\end{array}$ & Coorte & $\begin{array}{c}\text { Não foi encontrado forte } \\
\text { associação de métodos } \\
\text { contraceptivos e complicações } \\
\text { hipertensivas na gestação } \\
\text { (1,22 vezes maior do que } \\
\text { nas usuárias de método de } \\
\text { barreira). Sugere-se maiores } \\
\text { estudos sobre o assunto para } \\
\text { elucidação dos dados. }\end{array}$ \\
\hline $\begin{array}{l}\text { Lanza et al. } \\
(2013)^{18}\end{array}$ & $\begin{array}{c}312.395 \text { mulheres } \\
\text { e } 11.822 \text { casos } \\
\text { de fratura }\end{array}$ & $\begin{array}{l}\text { Determinar se o uso de } \\
\text { medroxiprogesterona } \\
\text { injetável aumenta o risco } \\
\text { de fratura em usuárias }\end{array}$ & $\begin{array}{c}\text { Coorte } \\
\text { retrospectivo }\end{array}$ & $\begin{array}{c}\text { As usuárias de } \\
\text { medroxiprogesterona } \\
\text { tiveram maior número de } \\
\text { casos de fratura, mas não } \\
\text { encontrou-se associação do } \\
\text { uso da medicação aos casos } \\
\text { encontrados }\end{array}$ \\
\hline $\begin{array}{l}\text { Longatto- } \\
\text { Filho et al. } \\
(2011)^{22}\end{array}$ & $\begin{array}{c}12 \text { mil mulheres } \\
\text { no Brasil e } \\
\text { Argentina }\end{array}$ & $\begin{array}{l}\text { Avaliar o risco de HPV, } \\
\text { NIC e câncer cervical } \\
\text { em usuárias de métodos } \\
\text { anticoncepcionais }\end{array}$ & Coorte & $\begin{array}{c}\text { Não foram encontradas } \\
\text { evidências de associação } \\
\text { de contracepção hormonal e } \\
\text { risco de infecção por HPV ou } \\
\text { NIC nessa coorte }\end{array}$ \\
\hline $\begin{array}{c}\text { Heinemann et al. } \\
(2010)^{23}\end{array}$ & $\begin{array}{c}451 \text { casos } \\
\text { de trombose } \\
\text { venosa profunda } \\
\text { e } 1920 \text { controles }\end{array}$ & $\begin{array}{c}\text { Investigar se os } \\
\text { anticoncepcionais } \\
\text { orais combinados com } \\
\text { gestodeno apresentam } \\
\text { maior risco de troboembolia }\end{array}$ & Caso-controle & $\begin{array}{c}\text { Não foi observado maior risco } \\
\text { de eventos trombóticos entre } \\
\text { as usuárias de contraceptivos } \\
\text { contendo gestodeno }\end{array}$ \\
\hline
\end{tabular}

adolescente e necessidade de avaliação de critérios de elegibilidade de métodos e acompanhamento seguros.

Historicamente discutido, o tema da associação entre o uso de contraceptivos hormonais e sua possibilidade de associação com câncer de mama foi estudado na Europa, uma uma coorte de 1.797.932 mulheres foram acompanhadas para observação do desenvolvimento da patologia e o uso ou não de métodos hormonais ${ }^{8}$. Pesquisadores concluíram que uso de hormônios, além de estarem associados ao desenvolvimento de câncer de mama na amostra observada, o risco não apareceu como menor nas mulheres que descontinuaram o uso após longo período de tratamento. Além disso, observou-se que o tempo de exposição influenciou no resultado desfavorável, o que vai ao encontro da presente revisão realizada.

\section{CONSIDERAÇÕES FINAIS}

Verificamos que o uso de anticoncepcionais hormonais é seguro e bem tolerado pela população em geral. Como qualquer terapêutica medicamentosa, pode estar associada a riscos. A indicação e utilização de dos mesmos por adolescentes apresentam, como na população em geral, riscos e benefícios evidenciados na literatura internacional. Considerando-se a fisiologia do crescimento e desenvolvimento ainda presentes na adolescência, tornam-se necessários cuidados e atenção na indicação do uso para essa população.

Poucos estudos abordam a questão através de pesquisas com delineamento sobre as necessidades e 
demandas exclusivamente da população adolescente, apresentando os resultados dentro de amostras generalizadas com mulheres de maior idade e demais condições fisiológicas. Contesta-se a afirmação de que o uso de tais métodos de forma precoce não envolva risco maiores a essa população sem a realização de delineamentos de pesquisa que aprofundem o conhecimento acerca da temática.

Todavia, o uso dessa terapêutica não pode ser descartada dadas as características da sexualidade que estão evidenciados nessa faixa etária. Porém sua indicação deve ser pautada em rigorosa avaliação individual e sob controle constante dos riscos associados ao seu uso. Fica evidente a associação de complicações vasculares associados aos contraceptivos hormonais, principalmente eventos trombóticos, nas populações descritas. A realização de acompanhamento de longo prazo, com estudos de coorte que avaliem tal relação em população exclusivamente adolescente, poderia elucidar o resultado com maior nível de evidência.
Ao contrário do pensamento de muitas décadas e observado no manejo dessa necessidade entre adolescentes, o uso do DIU não aparece como método de risco, ao contrário, pode ser pensado como estratégia de prevenção da gestação nessa faixa etária. Trata-se de um método de longo prazo, com seguro controle da liberação hormonal, evitando esquecimentos e podendo ser retirado conforme desejo da paciente.

Características distintas da população adolescente devem ser consideradas na tomada de decisão profissional, bem como de aconselhamento do método mais adequado a cada paciente. Maiores estudos sobre a temática são necessários para a elucidação de dúvidas a respeito da utilização e dos riscos a curto e longo prazo por essa população.

\section{Conflitos de Interesse}

Os autores declaram não ter conflitos de interesse.

\section{REFERÊNCIAS}

1. World Health Organization (WHO). Adolescents: health risks and solutions. Geneva: WHO; 2016. [citado 2018 jul 25]. Disponível em: http://www.who.int/mediacentre/ factsheets/fs345/en/

2. Brasil. Ministério da Saúde. Saúde sexual e saúde reprodutiva. Brasília: Ministério da Saúde, 2010. (Cadernos de Atenção Básica, n. 26) (Série A. Normas e Manuais Técnicos).

3. Oliveira RG. Blackbook enfermagem. Belo Horizonte: Ed Blackbook; 2016.

4. Cleland J, Conde-Agudelo A, Peterson $\mathrm{H}$, Ross J, Tsui A. Contraception and health. Lancet. 2012;380(9837):14956. http://dx.doi.org/10.1016/S01406736(12)60609-6. PMid:22784533.

5. Brasil. Ministério da Saúde. Manual de atenção à saúde dos adolescente. Brasília: Ministério da Saúde; 2008. [citado 2018 jul 25]. Disponível em http://bvsms.saude.gov.br/bvs/ publicacoes/saude_adolescente_ competencias_habilidades.pdf

6. Freitas F, Menke CH, Rivoire WA, Passos EP. Rotinas em ginecologia. 6 . ed. Porto Alegre: Artes Médicas; 2011.

7. Zettermark S, Perez Vicente R, Merlo $J$. Hormonal contraception increases the risk of psychotropic drug use in adolescent girls but not in adults: $\mathrm{A}$ pharmacoepidemiological study on 800000 Swedish women. PLoS One. 2018;13(3):e0194773. http://dx.doi. org/10.1371/journal.pone.0194773. PMid:29566064.

8. Morch LS, Skovlund CW, Hannaford PC, Iversen L, Fielding S, Lidegaard O. Contemporary hormonal contraception and the risk of breast cancer. $N$ Engl J Med. 2017;377(23):2228-39. http://dx.doi. org/10.1056/NEJMoa1700732. PMid:29211679.

9. Tepper NK, Dragoman MV, Gaffield ME, Curtis KM. Nonoral combined hormonal contraceptives and thromboembolism: a systematic review. Contraception. 2017;95(2):1309. http://dx.doi.org/10.1016/j. contraception.2016.10.005. PMid:27771476.

10. Hatch EE, Hahn KA, Mikkelsen EM, Riis AH, Sorensen HT, Rothman $\mathrm{KJ}$, et al. Pre-gravid oral contraceptive use in relation to birth weight: a prospective cohort study. Eur $J$ Epidemiol. 2015;30(11):1199-208. http://dx.doi.org/10.1007/s10654-0150053-2. PMid:26076921.

11. Birgisson NE, Zhao Q, Secura GM, Madden T, Peipert JF. Positive testing for neisseria gonorrhoeae and chlamydia trachomatis and the risk of pelvic inflammatory disease in IUD users. $J$ Womens Health (Larchmt). 2015;24(5):354-9. http:// dx.doi.org/10.1089/jwh.2015.5190. PMid:25836384.

12. Zhang J, Li C, Zhao WH, Xi X, Cao SJ, Ping $\mathrm{H}$, et al. Association between levonorgestrel emergency contraception and the risk of ectopic pregnancy: a multicenter case-control study. Sci Rep. 2015;5(1):8487. http://dx.doi.org/10.1038/srep08487. PMid:25674909.

13. Morrison CS, Chen PL, Kwok C, Baeten JM, Brown J, Crook AM, et al. Hormonal contraception and the risk of HIV acquisition: an individual participant data meta-analysis. PLoS Med. 2015;12(1):e1001778. http://dx.doi.org/10.1371/journal. pmed.1001778. PMid:25612136.

14. Heinemann K, Reed S, Moehner S, Minh TD. Risk of uterine perforation with levonorgestrel-releasing and copper intrauterine devices in the European Active Surveillance Study on Intrauterine Devices. Contraception. 2015;91(4):2749. http://dx.doi.org/10.1016/j. contraception.2015.01.007. PMid:25601352.

15. Parahuleva MS, Burgazli M, Langanke E, Dörr O, Parviz B, Mericliler M, et al. Monocyte CD40 
expression in Young healthy female smokers and/or oral contraceptives users without additional risk factors for atherosclerosis. Thromb Res. 2015;135(2):260-6. http://dx.doi. org/10.1016/j.thromres.2014.11.035. PMid:25541031.

16. Jensen ET, Daniels JL, Stürmer T, Robinson WR, Williams CJ, Vejrup $\mathrm{K}$, et al. Hormonal contraceptive use before and after conception in relation to preterm birth and small for gestational age: an observational cohort study. BJOG. 2015;122(10):1349-61. http://dx.doi. org/10.1111/1471-0528.13114. PMid:25318662.

17. Farley KE, Huber LR, WarrenFindlow J, Ersek JL. The association between contraceptive use at the time of conception and hypertensive disorders during pregnancy: a retrospective cohort study of prams participants. Matern Child Health J. 2014;18(8):1779-85. http://dx.doi. org/10.1007/s10995-014-1447-6. PMid:24535145.

18. Lanza LL, McQuay LJ, Rothman KJ, Bone HG, Kaunitz AM, Harel Z, et al. Use of depot medroxyprogesterone acetate contraception and incidence of bone fracture. Obstet Gynecol. 2013;121(3):593600. http://dx.doi.org/10.1097/ AOG.0b013e318283d1a1. PMid:23635623.

19. Warholm L, Petersen KR, Ravn P. Combined oral contraceptives' influence on weight, body composition, height, and bone mineral density in girls younger than 18 years: a systematic review. Eur $J$ Contracept Reprod Health Care. 2012;17(4):245-53. http://dx.doi.or g/10.3109/13625187.2012.692411. PMid:22758931.

20. Gronich N, Lavi I, Rennert G. Higher risk of venous thrombosis associated with drospirenonecontaining oral contraceptives: a population-based cohort study. CMAJ. 2011;183(18):E1319-25. http:// dx.doi.org/10.1503/cmaj.110463. PMid:22065352.

21. Trenor CC 3RD, Chung RJ, Michelson AD, Neufeld EJ, Gordon CM, Laufer MR, et al. Hormonal contraception and thrombotic risk: a multidisciplinary approach. Pediatrics. 2011;127(2):347-57. http://dx.doi. org/10.1542/peds.2010-2221. PMid:21199853

22. Longatto-Filho A, Hammes LS, Sarian LO, Roteli-Martins C, Derchain SF, Eržen M, et al. Hormonal contraceptives and the length of their use are not independent risk factors for high-risk HPV infections or high-grade CIN. Gynecol Obstet Invest. 2011;71(2):93-103. http:// dx.doi.org/10.1159/000320742. PMid:21150159.

23. Heinemann LA, Dinger JC Assmann A, Minh TD. Use of oral contraceptives containing gestodene and risk of venous thromboembolism: outlook 10 years after the third-generation "pill scare".
Contraception. 2010;81(5):4017. http://dx.doi.org/10.1016/j. contraception.2009.12.014. PMid:20399946.

24. Austin H, Lally C, Benson JM, Whitsett C, Hooper WC, Key NS. Hormonal contraception, sickle cell trait, and risk for venous thromboembolism among African American women. Am J Obstet Gynecol. 2009;200(6):620.e1-3. http:// dx.doi.org/10.1016/j.ajog.2009.01.038. PMid:19306959.

25. Jellesen R, Strandberg-Larsen K, Jørgensen T, Olsen J, Thulstrup AM, Andersen AM. Maternal use of oral contraceptives and risk of fetal death. Paediatr Perinat Epidemiol. 2008;22(4):334-40. http://dx.doi.org/10.1111/j.13653016.2008.00942.x. PMid:18578746.

26. Du Y, Rosner BM, Knopf H, Schwarz S, Dören M, Scheidt-Nave C. Hormonal contraceptive use among adolescent girls in Germany in relation to health behavior and biological cardiovascular risk factors. J Adolesc Health. 2011;48(4):331-7. http://dx.doi. org/10.1016/j.jadohealth.2011.01.004. PMid:21402260.

27. Biason TP, Goldberg TB, Kurokawa CS, Moretto MR, Teixeira AS, Nunes HR. Low-dose combined oral contraceptive use is associated with lower bone mineral content variation in adolescents over a 1-year period. BMC Endocr Disord. 2015;15(1):15. http://dx.doi.org/10.1186/s12902-0150012-7. PMid:25990414.

Recebido: 25 jul, 2018 Aceito: 22 abr, 2019 